\title{
VIEWPOINT
}

\section{To Decompress or Not? An Expected Utility Inspired Approach To Shared decision-making For Supratentorial Ischemic Stroke}

\author{
Christos Lazaridis ${ }^{*}$ (I) and Ali Mansour \\ C 2021 Springer Science+Business Media, LLC, part of Springer Nature and Neurocritical Care Society
}

\begin{abstract}
Patients with large territorial supratentorial infarctions are at high risk of cerebral edema, increased intracranial pressure, tissue herniation and death. There is strong evidence supporting prompt decompressive craniectomy after large hemispheric ischemic stroke as a means to reduce mortality. Nevertheless, functional outcomes can vary significantly. Clinical trials have traditionally judged these outcomes by a priori dichotomization without taking into account individual patient and caregiver preferences. If these are not incorporated into shared decision-making, there are significant risks in both directions, i.e. producing outcomes that may be judged as unacceptable to survivors, or not offering life-saving treatments to patients that according to their own values could be beneficial. In the absence of decision aids, we explore insights from decision theory and propose an expected utility-inspired approach as a supplementary navigating tool in the decision-making process. Four patient case scenarios are discussed as a demonstration of using individualized rankings of outcome preferences, and deriving expected utilities for interventions such as decompressive craniectomy versus medical therapy. The ultimate aim of the suggested approach is to assure that patient values are elicited and incorporated, and possible range and nature of outcomes are discussed, and by attempting to connect best available means to patient individualized ends.
\end{abstract}

Keywords: Hemispheric stroke, Decision-making, Decompressive craniectomy, Expected utility, Modified Rankin

\section{Introduction}

Patients with large territorial supratentorial infarctions are at high risk of cerebral edema, tissue herniation and death. The pooled analysis of three randomized controlled trials (RCTs) demonstrated significant reduction in mortality when decompressive craniectomy (DC) was performed within $48 \mathrm{~h}$ of malignant middle cerebral artery (MCA) infarction in patients $<60$ years of age, with an absolute risk reduction in mortality of $50 \%$ at 12 months [1]. Nevertheless, persistent morbidity and disability are common, and individual preferences regarding the degree of treatment performed in

\footnotetext{
*Correspondence: lazaridis@uchicago.edu

Neurocritical Care Unit, Departments of Neurology, and Surgery (Section of Neurosurgery), University of Chicago Medical Center, 5841 S. Maryland
} Avenue, Chicago, IL, USA the face of severe brain injury must be considered. For patients $>60$ years of age, DC also significantly reduces mortality; however, functional independence is highly unlikely [2]. The 2019 update on Guidelines for the Early Management of Patients With Acute Ischemic Stroke recommends that discussion of care options including $\mathrm{DC}$ versus medical therapy alone (MT), should take place quickly with patients (if possible) and caregivers [3]. The goal is to include patient-centered preferences in shared decision-making, especially during prognosis formation and when considering interventions or limitations in treatments. However, the guidelines offer no specific insights on how these discussions should be structured, how the alternatives should be presented, and how the potential outcomes should be understood. Furthermore, no guidance is furnished on how patient values are to be weighed in on choosing among alternatives. 
In this article, we attempt offering a conceptual framework based on principles of rational decision-making and apply expected utility theory (EUT) to assist surrogates and clinicians to think through the available choices, discuss possible outcomes and incorporate patient preferences into shared decision-making deliberations in the setting of large territorial ischemic infarction. The plan of the article is as follows: the first methodological section describes principles of decision theory and specifically of EUT. The second section applies these theoretical insights to four possible patient scenarios based on different value preferences and of different ages, to demonstrate how the results may assist shared decision-making.

\section{Methods}

\section{Expected Utility Theory}

EUT is an account of how to choose rationally when one is not sure which outcome will result from one's acts. The core idea is to choose the act with the highest expected utility (EU). We here employ EUT as a normative theory-that is, a theory of how people should make decisions (in classical economics, EUT is often used as a descriptive or as a predictive theory). The expected utility of an act or choice is a weighted average of the utilities of each of its possible outcomes, where the utility of an outcome measures the extent to which that outcome is preferred, or preferable, to the alternatives [4]. The utility of each outcome is weighted according to the probability that the act will lead to that outcome; EUT provides a way of ranking options according to how choiceworthy they are: the higher the EU, the better it is to choose the act. Accordingly, the EU of a medical (or surgical) intervention A depends on two features: 1 . The patient derived evaluation of each possible outcome, measured by a real number called a utility. This value is to be assigned according to patient preferences either by directly eliciting or via surrogates, or based on previously expressed wishes. 2 . The probability of each outcome conditional on intervention A.

Given this, A's expected utility is defined as:

$$
\mathrm{EU}(\mathrm{A})=\sum_{\mathrm{o} \in \mathrm{O}} \mathrm{P}_{\mathrm{A}}(\mathrm{o}) \mathrm{U}(\mathrm{o})
$$

where $\mathrm{O}$ is the set of outcomes, $\mathrm{P}_{\mathrm{A}}(\mathrm{O})$ is the probability of outcome $\mathrm{O}$ conditional on $\mathrm{A}$ and $\mathrm{U}(\mathrm{O})$ is the utility of $\mathrm{O}$. The term $\mathrm{P}_{\mathrm{A}}(\mathrm{O})$ represents the probability of $\mathrm{O}$ given $\mathrm{A}-$ roughly, how likely it is that outcome $\mathrm{O}$ will occur, on the supposition that the agent chooses or undergoes intervention $\mathrm{A}$. The different outcomes for our case scenarios are the different scores in the modified Rankin Scale (mRS) as reported in the relevant RCTs [5]. The mRS scores range from 0 to 6: 0 indicating no symptoms at all, 1 indicating no significant disability despite symptoms, being able to carry out all usual duties and activities, 2 indicating slight disability, being unable to carry out all previous activities, but able to look after own affairs without assistance, 3 indicating moderate disability, requiring some help, but being able to walk without assistance, 4 indicating moderately severe disability, being unable to walk without assistance and unable to attend to own bodily needs without assistance, 5 indicating severe disability, being bedridden, incontinent, and requiring constant nursing care and attention, and 6 indicating death. The probabilities of obtaining these outcomes will refer to the statistical frequencies of different mRS scores as observed in the aforementioned RCTs (see reference 1 for patients $<60$, and reference 2 for patients $>60$ years of age).

The term $\mathrm{U}(\mathrm{O})$ represents the utility of a certain outcome $\mathrm{O}$-roughly, how valuable $\mathrm{O}$ is according to patient's preferences. Formally, $\mathrm{U}$ is a function that assigns a real number to each of the outcomes (the units associated with $U$ are typically called utiles, so that if $\mathrm{U}(\mathrm{O})=2$, we say that $\mathrm{O}$ is worth 2 utiles); the greater the utility, the more valuable or more preferred the outcome. Assigning utiles to different mRS categories provides the opportunity to patients or surrogates to express their subjective evaluations on how preferable or acceptable these functional outcomes would be for them. It follows that the relevant utiles for our examples will come from preference rankings of mRS scores; as a reference, we are assigning 100 utiles to a mRS of zero. It will be up to individual patients/surrogates to assign utiles to the different mRS categories. A utile of zero signifies that this outcome is to be avoided in as strict a fashion as numerically quantifiable.

\section{Calculation of Expected Utility}

The expected utility is equal to the EU of all outcomes for a particular intervention. It is derived by weighting the utility of each possible outcome by its probability. For intervention A with 1 to $\mathrm{x}$ outcomes and probabilities $\mathrm{P}$, it follows:

$\mathrm{EU}(\mathrm{A})=\mathrm{P}_{\mathrm{o} 1} \cdot \mathrm{U}_{\mathrm{o} 1}+\mathrm{P}_{\mathrm{o} 2} \cdot \mathrm{U}_{\mathrm{o} 2}+\ldots \mathrm{P}_{\mathrm{ox}} \cdot \mathrm{U}_{\mathrm{ox}}$. Similarly for intervention $\mathrm{B}, \mathrm{EU}(\mathrm{B})$ can be calculated. This then directly allows to compare $\mathrm{EU}(\mathrm{A})$ with $\mathrm{EU}(\mathrm{B})$.

\section{Case Scenarios}

For demonstration purposes, four case scenarios follow where the focus of shared decision-making is DC vs. MT. 
For patients $<60$ years of age, the 12 -month probabilities associated with the two interventions are taken from the pooled analysis of the three European RCTs (reference 1), whereas for $>60$, from the DESTINY II trial (reference 2).

\section{Case A}

Fifty-five years old with the following utility ranking: mRS 2 (100), mRS 3 (70), mRS 4 (10), mRS 5 and 6 (0). Notice the precipitous drop from mRS 3 to 4 and that $\mathrm{mRS} 4$ is only marginally preferred from mRS 5 and 6 .

\section{Utility Function Curve}

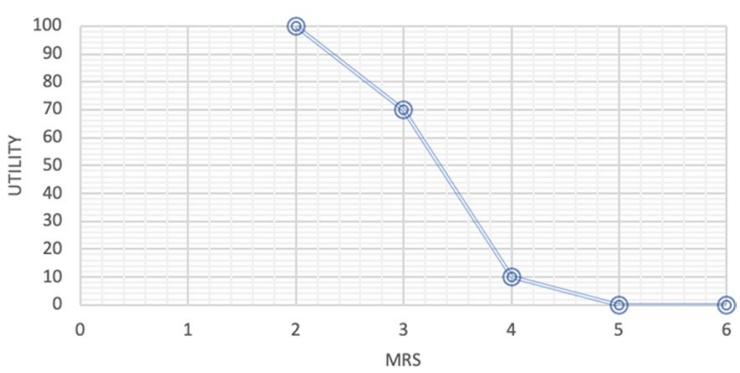

\begin{tabular}{|llll}
\hline mRS & Utility ranking & $\begin{array}{l}\text { Probability for mRS } \\
\text { at } 12 \text { months } \\
\text { with DC }\end{array}$ & $\begin{array}{l}\text { Probability for mRS } \\
\text { at } 12 \text { months with MT }\end{array}$ \\
\hline 2 & 100 & 0.14 & 0.02 \\
3 & 70 & 0.29 & 0.19 \\
4 & 10 & 0.31 & 0.02 \\
5 & 0 & 0.04 & 0.05 \\
6 & 0 & 0.22 & 0.71 \\
\hline
\end{tabular}

$\mathrm{EU}(\mathrm{DC})=0.14(100)+0.29(70)+0.31(10)+0.04(0)+$ $0.22(0)=37.4 . \mathrm{EU}(\mathrm{MT})=0.02(100)+0.19(70)+0.02(10)$ $+0.05(0)+0.71(0)=15.5 . \quad \mathrm{EU}(\mathrm{DC})>\mathrm{EU}(\mathrm{MT}) \quad$ suggesting that $\mathrm{DC}$ is the intervention most likely to confer outcomes consistent with A's values. This is an example where generically dichotomizing outcomes mRS $0-4$ as favourable would be inconsistent with this patient's evaluation.

\section{Case B}

Fifty-two years old, highly independent and against the idea of surviving with any disability that would prevent full return to all pre-morbid duties and activities: mRS 2 (20), mRS $3(0)$, mRS $4(0)$, mRS $5(0)$, mRS $6(10)$. This is a ranking example where moderate and severe disability states are ranked worse than death as outcomes.

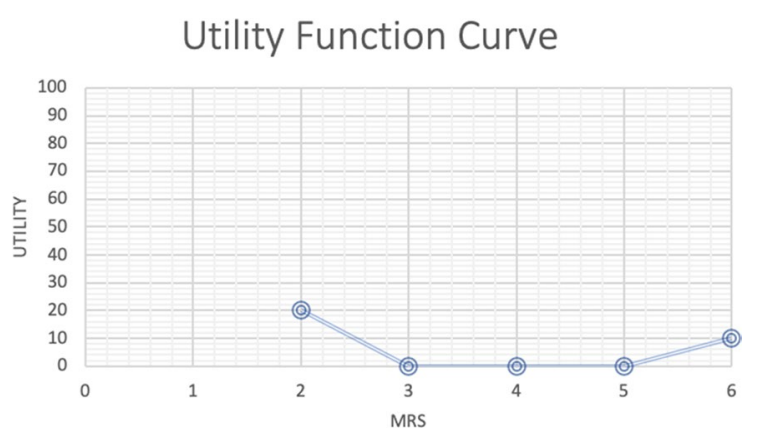

\begin{tabular}{llll} 
mRS & Utility ranking & $\begin{array}{l}\text { Prob- } \\
\text { ability for } m R S S \\
\text { at } 12 \text { months } \\
\text { with DC }\end{array}$ & $\begin{array}{l}\text { Prob- } \\
\text { ability for } m R S \\
\text { at } 12 \text { months } \\
\text { with MT }\end{array}$ \\
\hline 2 & 20 & 0.14 & 0.02 \\
3 & 0 & 0.29 & 0.19 \\
4 & 0 & 0.31 & 0.02 \\
5 & 0 & 0.04 & 0.05 \\
6 & 10 & 0.22 & 0.71 \\
\hline
\end{tabular}

$\mathrm{EU}(\mathrm{DC})=0.14(20)+0.29(0)+0.31(0)+0.04(0)+0.22$ $(10)=5 . \mathrm{EU}(\mathrm{MT})=0.02(20)+0.19(0)+0.02(0)+0.05(0)$ $+0.71(10)=7.5$. $\mathrm{EU}(\mathrm{DC})<\mathrm{EU}(\mathrm{MT})$ suggesting that $\mathrm{DC}$ should not be offered to $B$.

\section{Case C}

Forty-four years old with the following utility ranking mRS 2 (80), mRS 3 (40), mRS 4 (20), mRS 5 (0), mRS 6 (0).

\section{Utility Function Curve}

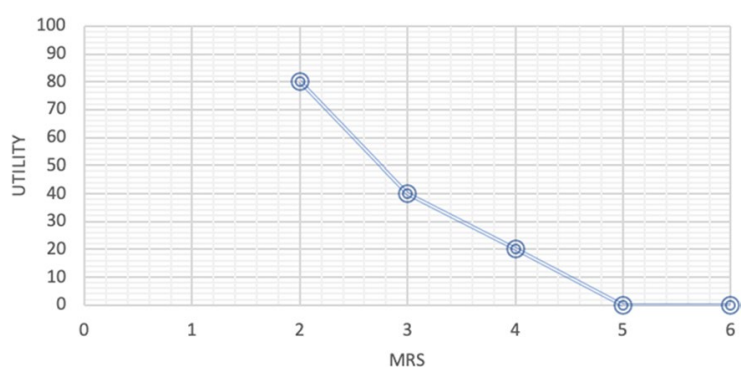




\begin{tabular}{llll}
\hline mRS & Utility ranking & $\begin{array}{l}\text { Prob- } \\
\text { ability for mRS } \\
\text { at } 12 \text { months } \\
\text { with DC }\end{array}$ & $\begin{array}{l}\text { Prob- } \\
\text { ability for } m R S \\
\text { at } 12 \text { months } \\
\text { with MT }\end{array}$ \\
\hline 2 & 80 & 0.14 & 0.02 \\
3 & 40 & 0.29 & 0.19 \\
4 & 20 & 0.31 & 0.02 \\
5 & 0 & 0.04 & 0.05 \\
6 & 0 & 0.22 & 0.71 \\
\hline
\end{tabular}

$\mathrm{EU}(\mathrm{DC})=0.14(80)+0.29(40)+0.31(20)+0.04(0)+0.2(0)$ $=29$. $\mathrm{EU}(\mathrm{MT})=0.02(80)+0.19(40)+0.02(20)+0.05(0)+$ $0.71(0)=9.6$. $E U(D C)>E U(M T)$ suggesting that $D C$ is the intervention most likely to confer outcomes consis tent with C's values.

\section{Case D}

Seventy-four years old, with utmost value the preservation of life; mRS 3 (80), mRS 4 (60), mRS 5 (40), mRS 6 (0).

\section{Utility Function Curve}

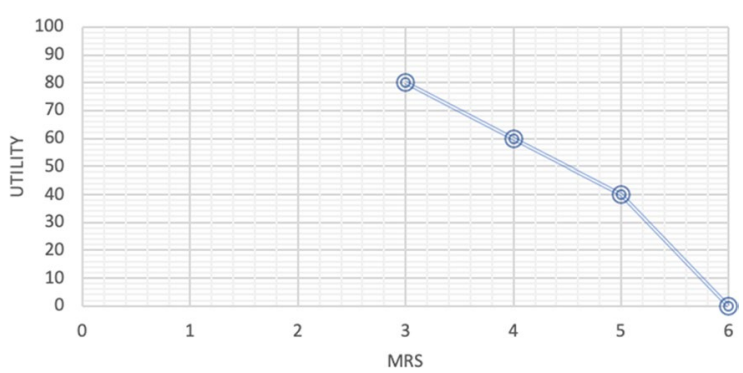

\begin{tabular}{llll}
\hline mRS & Utility ranking & $\begin{array}{l}\text { Probability for mRS } \\
\text { at } 12 \text { months } \\
\text { with DC }\end{array}$ & $\begin{array}{l}\text { Probability for mRS } \\
\text { at } 12 \text { months with MT }\end{array}$ \\
\hline 3 & 80 & 0.06 & 0.05 \\
4 & 60 & 0.32 & 0.11 \\
5 & 40 & 0.19 & 0.08 \\
6 & 0 & 0.43 & 0.76 \\
\hline
\end{tabular}

$\mathrm{EU}(\mathrm{DC})=0.06(80)+0.32(60)+0.19(40)+0.43(0)$ $=31.6 . \mathrm{EU}(\mathrm{MT})=0.05(80)+0.11(60)+0.08(40)$ $+0.76(0)=13.8$. EU(DC) $>\mathrm{EU}(\mathrm{MT})$ suggesting that $\mathrm{DC}$ is the recommended approach as a means to D's values and wishes.

\section{Discussion}

The 2019 update on Guidelines for the Early Management of Patients With Acute Ischemic Stroke provides a moderate strength (level IIA) recommendation supporting DC for patients $\leq 60$ years of age who deteriorate neurologically within $48 \mathrm{~h}$ from brain swelling associated with unilateral MCA infarctions despite medical therapy. This recommendation is based on the pooled analysis of 3 RCTs concluding that "decompressive surgery undertaken $48 \mathrm{~h}$ of stroke onset reduces mortality and increases the number of patients with a favorable functional outcome" [1]. However, it is important to note that favourable outcome was defined by the trial investigators as a mRS score of 0 to 4 . According to such a dichotomization, favourable outcome includes being unable to walk unaided and requiring assistance to attend to bodily needs. Beyond questioning the investigators choice (versus the more traditional 0-3 mRS as favourable), we need to ask "favorable for whom?" [6, 7]. It only seems appropriate that the arbitration and evaluation of an outcome should be primarily coming from patients and caregivers. The decision to offer a life-saving treatment after a large hemispheric infarction has to be balanced against great uncertainty in regards to functional outcomes and quality of survival; it is to be made in conjunction with surrogate decision makers via shared decision-making for establishing patient-specific goals [8]. In this manuscript, we suggest an approach inspired by principles of normative theories of rational choice, and specifically of expected utility theory. In practice, this would involve discussing in detail the possible outcomes using as a reference the descriptions of the different mRS scores. Then we would need to understand the patient's preferences and as possible to create a utility ranking of comparative preferences. The final step involves calculating expected utilities for the different interventions in order to get a sense of which intervention is the best means to the particular patient's needs and values.

There are multiple nuances to shared decision-making; clinicians should be aware of cognitive biases (affecting clinicians and surrogates) that may operate at an unconscious level yet may influence behavior and potentially the care provided $[9,10]$. Cognitive biases and heuristics can affect decision-making by distorting the understanding of the nature of a certain choice or decision and the foreseeable consequences $[11,12]$. A decision-theoretical model could shield against biases and enhance decision-making by providing a method to rank available choices according to patient-specific values. An expected utility theoryinspired approach can play the role of a decision procedure or aid when patients, surrogates, and clinicians are uncertain of the consequences among available interventions.

\section{Limitations}

The presented model purports to offer a "formula" for guiding decision-making based on ranking of outcomes according to patient values, together with probabilistic information of alternative interventions associated with these outcomes. However, we are not suggesting replacing 
in depth discussions with surrogates (and patients where feasible) with a simple algorithmic calculus. The process of deriving EUs for the different interventions can only be helpful if it is based on understanding, as best as possible, what kind(s) of survival would a patient consider preferable or acceptable, and what outcomes would this patient consider unacceptable or a disservice. It is crucial to consider that currently there are no decision aids that could assist surrogates and clinicians in rationally navigating available options, describing the nature and range of outcomes, and incorporating patients' values into goals of care. Additional limitations are the ones inherited by using the mRS; the scale lacks detail and granularity in terms of the described outcomes. Specific neurologic deficits and potential for recovery are not part of the scale. These are salient considerations and have to be part of careful discussion with patients and families. A further implication is that we need to expand the way we evaluate outcomes towards multiple dimensions that describe not only the nature and degree of deficits, and disability, but various aspects of well-being after large hemispheric stroke. Finally, it may seem unrealistic to ask surrogates, under the pressure of a goals-of-care meeting, to come up with a scored ranking of preferences; it actually may not be possible or advisable in situations of great urgency (for an EUT-guided approach that does not use ordinal ranking but only focuses on pivotal decision points see reference 13). The overall approach needs to be assessed prospectively in a pilot feasibility study, and it would be an important next step in evaluating the theoretical merits of the method. Such an evaluation would also have to be sensitive towards cultural norms of communication and shared-decision-making preferences. Nevertheless, one should also consider that we do not currently have data on how these conversations are conducted, how and if the range of outcomes is discussed, on the quality of information provided by clinicians, and on how this information is perceived and understood by patients and surrogates. Such data are necessary if we are to improve upon how we conduct shared decision-making. In this vein, it would be also important to test rates of retrospective consent to DC with pre-procedural outcome evaluations. The suggested model is meant to be supplementary to such critical aspects not as a substitute.

\section{Conclusions}

There is strong evidence supporting prompt decompressive craniectomy after large hemispheric ischemic stroke as a means to reduce mortality. Nevertheless, functional outcomes can vary significantly. Clinical trials have traditionally judged these outcomes by a priori dichotomization without taking into account individual patient and caregiver preferences. If these are not incorporated into shared decision-making, there are significant risks in both directions, i.e. producing outcomes that may be judged as unacceptable to survivors, or not offering life-saving treatments to patients who according to their own values could be beneficial. In the absence of decision aids, we propose an expected utility theory-inspired approach as a supplementary tool in the decision-making process, in order to assure that patient values are elicited and incorporated, the possible range and nature of outcomes are discussed, and by attempting to connect best available means to patient individualized ends.

\section{Sources of Funding}

$\mathrm{CL}$ acknowledges support from the Stevanovich Institute on the Formation of Knowledge (SIFK) at the University of Chicago; this manuscript is part of work based on a SIFK Faculty Research Grant on Shared decision-making in Neurocritical Care.

\section{Publisher's Note}

Springer Nature remains neutral with regard to jurisdictional claims in published maps and institutional affiliations.

Received: 31 October 2020 Accepted: 21 January 2021

Published online: 18 February 2021

\section{References}

1. Vahedi K, Hofmeijer J, Juettler E, et al. DECIMAL, DESTINY, and HAMLET Investigators. Early decompressive surgery in malignant infarction of the middle cerebral artery: a pooled analysis of three randomized controlled trials. Lancet Neurol. 2007;6:215-22.

2. Jüttler E, Unterberg A, Woitzik J, et al. DESTINY II Investigators. Hemicraniectomy in older patients with extensive middle-cerebral-artery stroke. N Engl J Med. 2014:370:1091-100.

3. Powers WJ, Rabinstein AA, Ackerson T, et al. Stroke. 2019;50(12):e344-418.

4. Briggs RA, Normative Theories of Rational Choice: Expected Utility, The Stanford Encyclopedia of Philosophy (Fall 2019 Edition), Edward N. Zalta (ed.), $U R L=<$ https://plato.stanford.edu/archives/fall2019/entries/rationalit y-normative-utility/>.

5. van Swieten JC, Koudstaal PJ, Visser MC, Schouten HJ, van Gijn J. Interobserver agreement for the assessment of handicap in stroke patients. Stroke. 1988;19:604-7.

6. Puetz V, Campos CR, Eliasziw M, Hill MD, Demchuk AM. Calgary Stroke Program. Assessing the benefits of hemicraniectomy: what is a favourable outcome? Lancet Neurol. 2007;6(3):580-1.

7. Honeybul S, Ho KM, Blacker DW. ORACLE stroke study: opinion regarding acceptable outcome following decompressive hemicraniectomy for ischemic stroke. Neurosurgery. 2016;79(2):231-6.

8. Lazaridis C. End-of-life considerations and shared decision making in neurocritical care. Continuum. 2018;24:1794-9.

9. Rohaut B, Claassen J. Decision making in perceived devastating brain injury: a call to explore the impact of cognitive biases. Br J Anaesth. 2018;120:5-9.

10. Lazaridis C. Withdrawal of life-sustaining treatments in perceived devastating brain injury: the key role of uncertainty. Neurocrit Care. 2019:30:33-41.

11. Blumenthal-Barby JS, Krieger H. Cognitive biases and heuristics in medical decision-making: a critical review using a systematic search strategy. Med Decis Making. 2015;35:539-57.

12. Blumenthal-Barby JS. Biases and heuristics in decision-making and their impact on autonomy. Am J Bioeth. 2016;16:5-15.

13. Lazaridis C. Deciding Under Uncertainty: The Case of Refractory Intracranial Hypertension. Front Neurol. 2020;20(11):908. 